\title{
Platinum Metal Complexes of Carbaboranylphophines: Potential Anti Cancer Agents
}

\author{
Ilham Maulana ${ }^{1} *$, Peter Loennecke ${ }^{2}$, Evamarie Hey-Hawkins ${ }^{2}$ \\ ${ }^{1}$ Department of Chemistry, Faculty of Mathematics and Natural Science, Syiah Kuala University, Banda Aceh, \\ Indonesia, Email: ilham.maulana@gmail.com \\ ${ }^{2}$ Institute of Inorganic Chemistry, Leipzig University, Leipzig, Germany
}

\begin{abstract}
Polyhedral heteroboranes in particular dicarba-closo-dodecaboranes(I2) and their organic derivatives have been the subject of intense research for over 40 years due to their unique chemical and physical properties. The initial attraction to dicarba-closododecaboranes(12) In the medicinal chemistry research, was a result of their high boron content and stability to catabolism, which are important criteria for cancer therapy, such as BNCT (boron neutron capture therapy) agents. The coordination compounds of the platinum group metals have also received large interest for their potential application as chemotherapeutic agents, since cis-diamminedichloroplatinum(II), cisplatin, has been reported to have capability as tumor inhibitor. Hence, applications can be envisioned for related cis platinum complexes. Complex of cis-rac- $\left[\mathrm{PtCl}_{2}\left\{\mathrm{I}, 2-(\mathrm{PRCl})_{2} \mathrm{C}_{2} \mathrm{~B}_{10} \mathrm{H}_{10}\right\}\right]\left(\mathrm{R}=\mathrm{Ph},{ }^{t} \mathrm{Bu}, \mathrm{NEt} \mathrm{t}_{2}, \mathrm{NPh}_{2}\right)$ have been synthesized by employing known carbaborane based phosphine ligands of clorophoshino-closo-dodecaborane, with complex of cis-[PtCl2(COD)] (COD $=1,5$ cyclooctadiene) in an $\mathrm{N}_{2}$-atmosphere. The obtained complexes possess expected structure configuration, namely cis-rac. The characterization of the complex has been carried out using IH, 3IP, I3C and IIB-NMR (Nuclear Magnetic Resonance), X-ray of single crystals, elemental analysis, IR (infra red) and mass spectroscopy (MS). The ${ }^{3} I P\{I H\} N M R$ spectra of all the platinum complexes distinctly show the typical platinum satellites which are attributed to $31 \mathrm{P}$ ${ }_{195} \mathrm{Pt}$-coupling, in which the ${ }^{3} \mathrm{P}\{\mathrm{I} \mathrm{H}\}$ NMR spectrum exhibits three lines with an intensity ratio of ca. I:4:I. The structure of the platinum complexes consists of a slightly distorted square-planar coordination sphere, in which the platinum atom is bonded to two chlorides and two phosphorus atoms of the chelating carbaboranylphosphine. Thus the platinum atoms exhibit the coordination number four, which is preferred in platinum(II) complexes.
\end{abstract}

Keywords: Platinum complexes, phosphine ligand, carbaborane

\section{INTRODUCTION}

Polyhedral heteroboranes in particular dicarba-closo-dodecaboranes(12) and their organic derivatives have been the subject of intense research for over 40 years due to their unique chemical and physical properties [Valliant, et al., 2002; Williams, 1992; Leites, 1992]. Thus, these compounds have been employed as catalysts [Yinghuai, et al., 2004; Larsen, et al., 2000; Teixidor, et al., 1996; Longato and Bresadola, 1982], as doping reagents in semiconductor materials [Bakun, et al., USSR Patent, 402241], and also in medical areas [Hawthorne, 1993; Soloway, 1998]. In the medicinal chemistry research, the initial attraction to dicarba-closododecaboranes(12) was a result of their high boron content and stability to catabolism, which are important criteria for BNCT (boron neutron capture therapy) agents. BNCT is based on the ${ }^{10} \mathrm{~B}(\mathrm{n}, \alpha)^{7} \mathrm{Li}$ reaction, which occurs when boron10 , which has a large capture cross section relative to the more abundant endogenous nuclei $\left({ }^{1} \mathrm{H},{ }^{12} \mathrm{C}\right.$, $\left.{ }^{31} \mathrm{P},{ }^{14} \mathrm{~N}\right)$, is exposed to thermal neutrons. The reaction releases high linear-energy-transfer radiation particles consisting of $\alpha$-particles $\left({ }^{4} \mathrm{He}\right)$ and lithium atoms ( $\left.{ }^{7} \mathrm{Li}\right)$ [Locher, 1936]. These particles cause direct DNA damage and tumor cell death.

*Corresponding author e-mail : ilham.maulana@gmail.com 
The coordination compounds of the platinum group metals have also received large interest for their potential application as chemotherapeutic agents, since cisdiamminedichloroplatinum (II), cisplatin [Peyrone, M., 1844], has been reported to have capability as tumor inhibitor [Rosenberg, et al., 1969]. Cisplatin is one of the most commonly used anti-cancer drugs today. It is used mainly in combination chemotherapy and effective against testicular carcinomas [Rozencweig, et al., 1987]. The excellent activity of cisplatin in testicular carcinomas has demonstrated the possibility to find new drugs in the area of inorganic chemistry that are capable of curing specific types of tumors. Numerous other metal compounds containing platinum, other platinum group metals, and even non-platinum metals were then shown to be effective against human tumors and tumors in animals [Kopf-Maier, 1994]. Hence, applications can be envisioned for related carbaboranylphosphines and transition metal complexes thereof.

In connection with our interest, related to the above mentioned areas, this paper describes the synthesis and the coordination properties of bidentate chiral tertiary phosphinodicarba-closododecaborane(12) ligands to form cis-platinum complexes. A range of ligands with different electronic and steric properties was synthesized by varying the substituents on the phosphorus atoms. Variation of these substituents can give significant changes in the electron-donor or -acceptor properties of the phosphines and in the steric demand of the phosphines as well. The resulting phosphines were employed as ligands in complexes of platinum metal.

\section{METHODS}

All the reactions were carried out in an atmosphere of dry nitrogen using standard Schlenk or vacuum line techniques. The solvents were purified (diethyl ether, THF, toluene: reflux over $\mathrm{Na} /$ benzophenone; $\mathrm{CH}_{2} \mathrm{Cl}_{2}, n$-hexane, $\mathrm{MeOH}$ : reflux over powdered $\mathrm{CaH}_{2}$ ) and distilled under nitrogen. The glassware was oven-dried for several hours, assembled while hot, and cooled in a stream of dry nitrogen gas. The infrared spectra were recorded on a Perkin-Elmer System 2000 FT-IR spectrometer scanning between 400 and $4000 \mathrm{~cm}^{-1}$ using $\mathrm{KBr}$ disks. The ${ }^{1} \mathrm{H},{ }^{13} \mathrm{C},{ }^{31} \mathrm{P}$ and ${ }^{11} \mathrm{~B}$ NMR spectra were recorded on an AVANCE DRX 400 spectrometer (Bruker). The chemical shifts for the ${ }^{1} \mathrm{H}$ and ${ }^{13} \mathrm{C}$ NMR spectra are reported in parts per million (ppm) at $400.13 \mathrm{MHz}$ and $100.6 \mathrm{MHz}$ with tetramethylsilane as standard. The chemical shifts for the ${ }^{31} \mathrm{P}$ NMR spectra are reported in parts per million (ppm) at $161.97 \mathrm{MHz}$ (with $85 \% \mathrm{H}_{3} \mathrm{PO}_{4}$ external standard) and chemical shifts for ${ }^{11} \mathrm{~B}$ NMR spectra are in parts per million (ppm) at $128.38 \mathrm{MHz}$ with $\mathrm{BF}_{3}\left(\mathrm{OEt}_{2}\right)$ as external standard. The mass spectra were recorded on an Ltd. ZABHSQ-VG Analytical Manchester Spectrometer (FAB mass spectra) and on an FT-ICR-MS Bruker-Daltonics ESI mass spectrometer (APEX II, 7 Tesla). The elemental analyses were recorded on a VARIO EL (Heraeus). The melting points were determined in sealed capillaries and are uncorrected. The products of the catalytic reactions were determined by GC analysis using an AGILENT 6890 gas chromatograph with split/splitless injector and a CP-CHIRASIL-LVAL column. The crystallographic data were collected on a Siemens CCD (SMART) diffractometer (compounds 5 and 6) and a StoeIPDS imaging plate diffractometer (compound 7). The empirical absorption correction was performed with SADABS [Sheldrick, 1998] (Siemens CCD diffractometer) and the numerical absorption correction using XRED (Stoe-IPDS diffractometer). The structures were solved by direct methods (SHELXTL PLUS) [SHELXTL PLUS, SHELXS, 1990].

The compound ortho-closo-carbaborane was donated by INEOS (A. N. Nesmeyanov Institute of Organoelement Compounds Russian Academy of Sciences, Moscow, Russian Federation). The chemicals $n$-butyllithium, dichlorophenylphosphine, $\mathrm{PCl}_{3}, N, N$-diethylamin, tert-butylchlorid, $N, N$-diphenylamin, COD $(1,5-$ cyclooctadiene) were used as purchased. $\mathrm{H}_{2}\left[\mathrm{PtCl}_{6}\right]$ - $6 \mathrm{H}_{2} \mathrm{O}$ was generously donated by Umicore.

${ }^{t} \mathrm{BuPCl}_{2}$ [Imori, 1992], $\mathrm{P}\left(\mathrm{NEt}_{2}\right) \mathrm{Cl}_{2}$ [Perich and Johns, 1988], $\mathrm{P}\left(\mathrm{NPh}_{2}\right) \mathrm{Cl}_{2}$ [Falius and Babin, 1976], [ $\mathrm{PtCl}_{2}$ (COD)] [Cotton, 1972; Brauer, G., 1981], were prepared according to the literature.

\section{Preparation of the Known Compounds}

rac-1,2-Bis(tert-butylchlorophosphino)-1,2dicarba-closo-dodecaborane(12) (1) [Sterzik et al., 2001], rac-1,2-bis(phenylchlorophosphino)-1,2dicarba-closo-dodecaborane(12) (2) [Balema et al., 1999; Alexander and Schroeder, 1963], rac-1,2Bis(N,N-diethylaminochlorophosphino)-1,2dicarba-closo-dodecaborane(12) (3), and rac-1,2Bis(N,N-diphenylaminochlorophosphino)-1,2dicarba-closo-dodecaborane(12) (4) [Stadlbauer et al., 2009] were prepared according to the literature and obtained in good yield. 


\section{Synthesis of cis-rac- $/ \mathrm{PtCl}_{2}\{1,2-$ $\left.\left.(P+B u C l)_{2} C_{2} B_{10} H_{10}\right\}\right](5)$}

A mixture of $0.2 \mathrm{~g} \quad\left(\begin{array}{lll}0.53 & \mathrm{mmol}\end{array}\right)$ $\left[\mathrm{PtCl}_{2}(\mathrm{COD})\right](\mathrm{COD}=1,5$-cyclooctadiene $), 0.21 \mathrm{~g}$ $(0.53 \mathrm{mmol}) 1$ and $50 \mathrm{ml}$ toluene was refluxed for $2 \mathrm{~h}$. The mixture was then concentrated to yield a white precipitate $(0.25 \mathrm{~g})$. Crystallization from toluene solution gave colorless crystals of $\mathbf{5}$. Yield: 0.25 g $(73 \%)$. M.p.: $280{ }^{\circ} \mathrm{C}$ (decomposes, turns brown). Found: $\mathrm{C} 18.70 ; \mathrm{H} 4.47 \%$. Calc. for $\mathrm{C}_{10} \mathrm{H}_{28} \mathrm{~B}_{10} \mathrm{Cl}_{4} \mathrm{P}_{2} \mathrm{Pt}$ : C $18.83 ; \mathrm{H} 4.31 \%$. FAB-MS, $m / z: 619\left(6 \%, M^{+}-\mathrm{Cl}\right), 584\left(2 \%, M^{+}-2 \mathrm{Cl}\right)$. Calc. for $\mathrm{C}_{10} \mathrm{H}_{28} \mathrm{~B}_{10} \mathrm{Cl}_{4} \mathrm{P}_{2} \mathrm{Pt}$ : $\mathrm{M}=655.25$. ${ }^{1} \mathrm{H}$ NMR $\left(\mathrm{CDCl}_{3} / \mathrm{TMS}\right.$, ppm): $3.75-1.93(\mathrm{~m}, \mathrm{vbr}, 10 \mathrm{H}$, $\left.\mathrm{C}_{2} \mathrm{~B}_{10} \mathrm{H}_{10}\right), 1.73\left(\mathrm{~d},{ }^{3} \mathrm{~J}_{\mathrm{PH}}=22 \mathrm{~Hz}, 18 \mathrm{H}, \mathrm{CH}_{3}\right) .{ }^{31} \mathrm{P}$ NMR $\left(\mathrm{CDCl}_{3}, \mathrm{ppm}\right): 122.6\left({ }^{1} J_{\mathrm{PPt}}=3873 \mathrm{~Hz}\right) \cdot{ }^{13} \mathrm{C}$ NMR ( $\left.\mathrm{CDCl}_{3} / \mathrm{TMS}, \mathrm{ppm}\right): 85.1\left(\mathrm{dt},{ }^{2} J_{\mathrm{CPt}}=102.6\right.$ $\left.\mathrm{Hz},{ }^{1} J_{\mathrm{PC}}=28.1 \mathrm{~Hz}, \mathrm{C}_{\text {cluster }}-\mathrm{P}\right), 50.1\left(\mathrm{~m},{ }^{2} J_{\mathrm{CPt}}=54.0\right.$ $\left.\mathrm{Hz},{ }^{1} J_{\mathrm{PC}}=40.1 \mathrm{~Hz},{ }^{3} J_{\mathrm{PC}}=7.9 \mathrm{~Hz}, \underline{\mathrm{CMe}_{3}}\right), 28.5(\mathrm{q}$, $\left.{ }^{1} J_{\mathrm{CH}}=130.0 \mathrm{~Hz},{ }^{2} J_{\mathrm{PC}}=4.5 \mathrm{~Hz}, \mathrm{CH}_{3}\right) .{ }^{11} \mathrm{~B} \mathrm{NMR}$ $\left(\mathrm{CDCl}_{3}, \mathrm{ppm}\right): 0.83\left(\mathrm{~d},{ }^{1} J_{\mathrm{BH}}=151 \mathrm{~Hz}, 2 \mathrm{~B}\right.$, $\left.\mathrm{C}_{2} \mathrm{~B}_{10} \mathrm{H}_{10}\right),-3.08\left(\mathrm{~d},{ }^{1} J_{\mathrm{BH}}=154 \mathrm{~Hz}, 2 \mathrm{~B}, \mathrm{C}_{2} \mathrm{~B}_{10} \mathrm{H}_{10}\right)$, $-9.44\left(\mathrm{~m}\right.$, vbr, 6B, $\left.\mathrm{C}_{2} \mathrm{~B}_{10} \mathrm{H}_{10}\right)$. IR $\left(\mathrm{KBr}, \mathrm{cm}^{-1}\right)$ : $3015 \mathrm{~m}, 2995 \mathrm{~m}, 2961 \mathrm{~m}, 2926 \mathrm{~m}, 2868 \mathrm{~m}(\mathrm{CH})$; 2678m, 2666s, 2639s, 2595s, 2576s, 2564s (BH); $1955 \mathrm{w}, 1626 \mathrm{w}, 1471 \mathrm{~s}, 1459 \mathrm{~s}, 1433 \mathrm{~m}, 1401 \mathrm{~s}$, $1368 \mathrm{~s}, 1261 \mathrm{~m}, 1165 \mathrm{~s}, 1070 \mathrm{~s}, 1017 \mathrm{~s}, 976 \mathrm{w}, 930 \mathrm{~m}$, 902w, 884w, 835m, 797s, 772m, 749s, 733s, $682 \mathrm{w}, 665 \mathrm{~m}, 629 \mathrm{~s}, 570 \mathrm{~s}, 542 \mathrm{~s}, 495 \mathrm{~s}, 458 \mathrm{~m}, 448 \mathrm{~m}$.

\section{Synthesis of cis-rac-/PtCl $2\{1,2-$ $\left.\left.(\mathrm{PPhCl})_{2} \mathrm{C}_{2} \mathrm{~B}_{10} \mathrm{H}_{10}\right\}\right]$ (6)}

A mixture of $0.2 \mathrm{~g} \quad(0.53 \mathrm{mmol})$ [ $\left.\mathrm{PtCl}_{2}(\mathrm{COD})\right], 0.23 \mathrm{~g}(0.53 \mathrm{mmol}) 2$ and $50 \mathrm{ml}$ toluene was refluxed for $2 \mathrm{~h}$. The mixture was then concentrated to yield $0.31 \mathrm{~g}$ of a white precipitate. Crystallization from toluene solution gave colorless crystals of $\mathbf{6}$. Yield: $0.31 \mathrm{~g}(83 \%)$. M.p.: $320{ }^{\circ} \mathrm{C}$ (decomposes, turns brown). Found: $\mathrm{C}$ $23.00 ; \mathrm{H} 2.06 \%$. Calc. for $\mathrm{C}_{14} \mathrm{H}_{20} \mathrm{~B}_{10} \mathrm{Cl}_{4} \mathrm{P}_{2} \mathrm{Pt}$ : C 24.19; H $2.90 \%$. FAB-MS, $m / z: 659\left(100 \%, M^{+}-\right.$ Cl), $624\left(14 \%, M^{+}-2 \mathrm{Cl}\right) 587\left(11 \%, M^{+}-3 \mathrm{Cl}\right)$. Calc. for $\mathrm{C}_{14} \mathrm{H}_{20} \mathrm{~B}_{10} \mathrm{Cl}_{4} \mathrm{P}_{2} \mathrm{Pt}: \mathrm{M}=695.25$. ${ }^{1} \mathrm{H}$ NMR $\left(\mathrm{C}_{6} \mathrm{D}_{6} / \mathrm{TMS}, \mathrm{ppm}\right): 7.70$ - $6.86(\mathrm{~m}, 10 \mathrm{H}, \mathrm{Ph}), 7.10$ 6.90 (several $\mathrm{m}, \mathrm{Ph}$ in $\left.\mathrm{C}_{7} \mathrm{H}_{8}\right), 3.73-1.01$ (m, vbr, $\left.10 \mathrm{H}, \mathrm{C}_{2} \mathrm{~B}_{10} \mathrm{H}_{10}\right), 2.10\left(\mathrm{~s}, 3 \mathrm{H}, \mathrm{CH}_{3}\right.$ in $\left.\mathrm{C}_{7} \mathrm{H}_{8}\right) .{ }^{31} \mathrm{P}$ NMR $\left(\mathrm{C}_{6} \mathrm{D}_{6}, \mathrm{ppm}\right): 97.8\left({ }^{1} J_{\mathrm{PPt}}=4038 \mathrm{~Hz}\right) .{ }^{11} \mathrm{~B}$ NMR $\left(\mathrm{C}_{6} \mathrm{D}_{6}, \mathrm{ppm}\right):-1.8\left(\mathrm{~d},{ }^{1} J_{\mathrm{BH}}=143 \mathrm{~Hz}, 4 \mathrm{~B}\right.$, $\left.\mathrm{C}_{2} \mathrm{~B}_{10} \mathrm{H}_{10}\right),-10.2\left(\mathrm{~d},{ }^{1} J_{\mathrm{BH}}=142 \mathrm{~Hz}, 4 \mathrm{~B}, \mathrm{C}_{2} \mathrm{~B}_{10} \mathrm{H}_{10}\right)$, $-13.6\left(\mathrm{~d},{ }^{1} J_{\mathrm{BH}}=168 \mathrm{~Hz}, 2 \mathrm{~B}, \mathrm{C}_{2} \mathrm{~B}_{10} \mathrm{H}_{10}\right)$. IR $(\mathrm{KBr}$, $\left.\mathrm{cm}^{-1}\right)$ : 3082w, 3055m, 3022m, 2959w, 2917w $(\mathrm{CH})$; 2614, $2582(\mathrm{BH}) ; 2191 \mathrm{w}, 1965 \mathrm{w}, 1894 \mathrm{w}$, $1809 \mathrm{w}(\mathrm{Ph}) ; 1603 \mathrm{w}, 1580 \mathrm{~m}, 1494 \mathrm{~m}, 1475 \mathrm{~m}$, $1436 \mathrm{~s}, 1384 \mathrm{w}, 1336 \mathrm{w}, 1310 \mathrm{~s}, 1283 \mathrm{w}, 1261 \mathrm{~m}$, $1186 \mathrm{~m}, 1160 \mathrm{~m}, 1097 \mathrm{~s}, 1076 \mathrm{~s}, 1025 \mathrm{~m}, 997 \mathrm{~m}$,
$981 \mathrm{~m}, 937 \mathrm{~m}, 900 \mathrm{~m}, 852 \mathrm{~s}, 798 \mathrm{~s}, 732 \mathrm{~s}, 713 \mathrm{~s}, 695 \mathrm{~s}$, 684s, 630s, 616s, 575s, 553s, 514s, 485s, 472s.

The low solubility of $\mathbf{6}$ prevents measurement of the ${ }^{13} \mathrm{C}$ NMR spectrum.

\section{Synthesis of cis-rac- $/ \mathrm{PtCl}_{2}\{\mathrm{I}, 2-$ $\left.\left\{P\left(\mathrm{NEt}_{2}\right) \mathrm{Cl}_{2} \mathrm{C}_{2} \mathrm{~B}_{10} \mathrm{H}_{10}\right\}\right](7)$ \\ A mixture of $0.14 \quad \mathrm{~g} \quad(0.37 \mathrm{mmol})$} [ $\left.\mathrm{PtCl}_{2}(\mathrm{COD})\right], 0.16 \mathrm{~g}(0.37 \mathrm{mmol}) 3$ and $50 \mathrm{ml}$ toluene was refluxed for $10 \mathrm{~h}$. The mixture was then concentrated to obtain $0.18 \mathrm{~g}$ of a white precipitate. Crystallization from toluene solution gave colorless crystals of 7. Yield: $0.18 \mathrm{~g}(70 \%)$. M.p.: $230{ }^{\circ} \mathrm{C}$ (decomposes, turns black). Found: $\mathrm{C}$ 19.7; $\mathrm{H}$ 4.43; $\mathrm{N} 3.43 \%$. Calc. for $\mathrm{C}_{10} \mathrm{H}_{30} \mathrm{~B}_{10} \mathrm{Cl}_{4} \mathrm{~N}_{2} \mathrm{P}_{2} \mathrm{Pt} \cdot 0.5 \mathrm{C}_{7} \mathrm{H}_{8}$ : C 22.17; H 4.69; N $3.83 \%$. FAB-MS, $m / z$ : $649\left(100 \%, M^{+}-\mathrm{Cl}\right)$, $614\left(81 \%, M^{+}-2 \mathrm{Cl}\right), 577\left(12 \%, M^{+}-3 \mathrm{Cl}\right), 542(8$ $\left.\%, M^{+}-4 \mathrm{Cl}\right)$. Calc. for $\mathrm{C}_{10} \mathrm{H}_{30} \mathrm{~B}_{10} \mathrm{Cl}_{4} \mathrm{~N}_{2} \mathrm{P}_{2} \mathrm{Pt}: \mathrm{M}=$ 685.31. ${ }^{1} \mathrm{H}$ NMR $\left(\mathrm{CDCl}_{3} / \mathrm{TMS}, \mathrm{ppm}\right): 3.65$ and $3.42\left(\mathrm{~m}, \mathrm{br}, 8 \mathrm{H}, \mathrm{CH}_{2}\right), 3.55-1.63(\mathrm{~m}, \mathrm{vbr}, 10 \mathrm{H}$, $\left.\mathrm{C}_{2} \mathrm{~B}_{10} \mathrm{H}_{10}\right), 1.23\left(\mathrm{t},{ }^{3} \mathrm{~J}_{\mathrm{HH}}=8 \mathrm{~Hz}, 12 \mathrm{H}, \mathrm{CH}_{3}\right) \cdot{ }^{31} \mathrm{P}$ NMR $\left(\mathrm{CDCl}_{3}, \mathrm{ppm}\right): 98.2\left({ }^{1} J_{\mathrm{PPt}}=4663 \mathrm{~Hz}\right) \cdot{ }^{13} \mathrm{C}$ NMR (CDCl $/$ TMS, ppm): 129.1 - 124.5 (several $\left.\mathrm{m}, \mathrm{C}_{7} \mathrm{H}_{8}\right), 90.8\left(\mathrm{tt},{ }^{1} J_{\mathrm{PC}}=25.2 \mathrm{~Hz},{ }^{2} J_{\mathrm{PC}}=25.2 \mathrm{~Hz}\right.$, $\left.{ }^{2} J_{\mathrm{CPt}}=168.8 \mathrm{~Hz}, \mathrm{C}_{\text {cluster }} \mathrm{P}\right), 44.3\left(\mathrm{~m}, \mathrm{vbr}, \mathrm{CH}_{2}\right)$, $21.5\left(\mathrm{q},{ }^{1} J_{\mathrm{CH}}=138.5 \mathrm{~Hz}\right) 12.8\left(\mathrm{q},{ }^{1} J_{\mathrm{CH}}=126.7 \mathrm{~Hz}\right.$, $\left.\mathrm{CH}_{3}\right) .{ }^{11} \mathrm{~B}$ NMR $\left(\mathrm{CDCl}_{3}, \mathrm{ppm}\right):-2.2\left(\mathrm{~d},{ }^{1} J_{\mathrm{BH}}=142\right.$ $\mathrm{Hz}, 4 \mathrm{~B}, \mathrm{C}_{2} \mathrm{~B}_{10} \mathrm{H}_{10}$ ), -10.2 (m, vbr, 4B, $\mathrm{C}_{2} \mathrm{~B}_{10} \mathrm{H}_{10}$ ), 14.0 (m, vbr, 2B, $\left.\mathrm{C}_{2} \mathrm{~B}_{10} \mathrm{H}_{10}\right)$. IR $\left(\mathrm{KBr}, \mathrm{cm}^{-1}\right)$ : 2981s, 2937s, 2894s (CH); 2621s, 2582s (BH); $1860 \mathrm{w}, 1706 \mathrm{w}, 1626 \mathrm{w}, 1494 \mathrm{w}, 1462 \mathrm{~m}, 1444 \mathrm{~m}$, $1382 \mathrm{~s}, 1363 \mathrm{~m}, 1343 \mathrm{~m}, 1289 \mathrm{~m}, 1262 \mathrm{w}, 1201 \mathrm{~s}$, $1151 \mathrm{~s}, 1100 \mathrm{~s}, 1076 \mathrm{~s}, 1057 \mathrm{~s}, 1020 \mathrm{~s}, 962 \mathrm{~s}, 925 \mathrm{w}$, 847s, 799s, 760w, 740m, 688m, 671m, 628s, 579s, 549s, 495s, 459m, 417w.

\section{Synthesis of cis-rac- $/ \mathrm{PtCl}_{2}\{\mathrm{I}, 2-$ $\left.\left.\left\{\mathrm{P}\left(\mathrm{NPh}_{2}\right) \mathrm{Cl}\right\}_{2} \mathrm{C}_{2} \mathrm{~B}_{10} \mathrm{H}_{10}\right\}\right](8)$}

A mixture of $0.13 \mathrm{~g}(0.35 \mathrm{mmol})$ [ $\left.\mathrm{PtCl}_{2}(\mathrm{COD})\right], 0.21 \mathrm{~g}(0.35 \mathrm{mmol}) 4$ and $35 \mathrm{ml}$ toluene was refluxed for $105 \mathrm{~h}$. The mixture was then filtrated and concentrated to obtain $0.043 \mathrm{~g}$ of a white precipitate of $\mathbf{8}$. Yield: $0.043 \mathrm{~g}(14 \%) .{ }^{1} \mathrm{H}$ NMR ( $\left.\mathrm{CDCl}_{3} / \mathrm{TMS}, \mathrm{ppm}\right): 7.76-7.26(\mathrm{~m}, \mathrm{br}, 20 \mathrm{H}$, $\mathrm{Ph}), 3.56$ - 1.53 (m, vbr, 10H, $\left.\mathrm{C}_{2} \mathrm{~B}_{10} \mathrm{H}_{10}\right) .{ }^{31} \mathrm{P}$ NMR $\left(\mathrm{CDCl}_{3}, \mathrm{ppm}\right): 94.6\left({ }^{1} J_{\mathrm{PPt}}=4895 \mathrm{~Hz}\right) .{ }^{11} \mathrm{~B} \mathrm{NMR}$ $\left(\mathrm{CDCl}_{3}, \mathrm{ppm}\right):-2.4\left(\mathrm{~m}, \mathrm{vbr}, 4 \mathrm{~B}, \mathrm{C}_{2} \mathrm{~B}_{10} \mathrm{H}_{10}\right),-11.2$ $\left(\mathrm{m}\right.$, vbr, $6 \mathrm{~B}, \mathrm{C}_{2} \mathrm{~B}_{10} \mathrm{H}_{10}$ ). The amount of substance obtained was insufficient for other characterization methods. 


\section{RESULTS AND DISCUSSION}

The preparation of a platinum complex with a non-chiral carbaboranylphosphine ligand, cis$\left[\mathrm{PtCl}_{2}\left\{1,2-\left(\mathrm{P}^{i} \mathrm{Pr}_{2}\right)_{2}-1,2-\mathrm{C}_{2} \mathrm{~B}_{10} \mathrm{H}_{10}\right\}\right]$ has been reported [Paavola et al., 2002], in which $\left[\mathrm{PtCl}_{2}(\mathrm{COD})\right]$ (COD = 1,5-cyclooctadiene) was used as starting material. Several platinum complexes with unsymmetrical tertiary bisphosphines of ortho-carbaborane were prepared as well, i. e. cis- $\left[\mathrm{PtCl}_{2}\left\{1-\mathrm{PPh}_{2}-2-\right.\right.$ $\left.\left.\mathrm{P}\left(\mathrm{NMe}_{2}\right)_{2}\left(\mathrm{C}_{2} \mathrm{~B}_{10} \mathrm{H}_{10}\right)\right\}\right], \quad$ cis- $\left[\mathrm{PtCl}_{2}\left\{1-\mathrm{PPh}_{2}-2-\right.\right.$ $\left.\left.\mathrm{P}\left(\mathrm{NMe}_{2}\right) \mathrm{F}\left(\mathrm{C}_{2} \mathrm{~B}_{10} \mathrm{H}_{10}\right)\right\}\right]$, cis-[ $\mathrm{PtCl}_{2}\left\{1-\mathrm{P}\left(\mathrm{NMe}_{2}\right) \mathrm{F}-2-\right.$ $\left.\left.\mathrm{P}\left(\mathrm{NMe}_{2}\right)_{2}\left(\mathrm{C}_{2} \mathrm{~B}_{10} \mathrm{H}_{10}\right)\right\}\right]$, and cis- $\left[\mathrm{PtCl}_{2}\left\{1-\mathrm{PPh}_{2}-2-\right.\right.$ $\left.\mathrm{PF}_{2}\left(\mathrm{C}_{2} \mathrm{~B}_{10} \mathrm{H}_{10}\right)\right\}$ ] [Hill et al., 1983]. However, platinum complexes with two chiral carbaboranylphosphine ligands were not yet explored.

\section{Synthesis and Spectroscopic Properties of Platinum Complexes with Carbaboranylphosphine Ligands}

The platinum complexes cis-rac- $\left[\mathrm{PtCl}_{2}\{1,2-\right.$ $\left.\left.(\mathrm{PRCl})_{2} \mathrm{C}_{2} \mathrm{~B}_{10} \mathrm{H}_{10}\right\}\right]\left(\mathrm{R}={ }^{t} \mathrm{Bu}(5), \mathrm{Ph}(6), \mathrm{NEt}_{2}(7)\right.$, $\mathrm{NPh}_{2}$ (8) were synthesized employing a similar procedure as for cis-[ $\mathrm{PtCl}_{2}\left\{1,2-\left(\mathrm{P}^{i} \mathrm{Pr}_{2}\right)_{2}-1,2-\right.$ $\left.\left.\mathrm{C}_{2} \mathrm{~B}_{10} \mathrm{H}_{10}\right\}\right]$, in which [ $\left.\mathrm{PtCl}_{2}(\mathrm{COD})\right]$ was stirred in a boiling toluene solution with a suitable chiral carbaboranylphosphine ligand (1-4).

Except

cis-rac-[ $\left[\mathrm{PtCl}_{2}\{1,2-\right.$ $\left.\left.\left\{\mathrm{P}\left(\mathrm{NPh}_{2}\right) \mathrm{Cl}\right\}_{2} \mathrm{C}_{2} \mathrm{~B}_{10} \mathrm{H}_{10}\right\}\right](8)$, all other platinum complexes were obtained in good yield (67-93\%). Complex 8 could only be obtained in $14 \%$ yield. The signal of the free ligand was observed in the ${ }^{31} \mathrm{P}$ NMR spectrum of the reaction mixture even after refluxing for more than $100 \mathrm{~h}$, while the amount of the side products increased. The steric demand of the bulkier substituent, $\mathrm{NPh}_{2}$, is presumably responsible for hampering the complexation. Compounds 5 - 8 were obtained as air- and water-stable solids that are slightly soluble in organic solvents.

The ${ }^{31} \mathrm{P}\left\{{ }^{1} \mathrm{H}\right\} \quad$ NMR spectra of all the platinum complexes distinctly show the typical platinum satellites which are attributed to ${ }^{31} \mathrm{P}-{ }^{195} \mathrm{Pt}$ coupling, in which the ${ }^{31} \mathrm{P}\left\{{ }^{1} \mathrm{H}\right\}$ NMR spectrum exhibits three lines with an intensity ratio of $c a$. 1:4:1 [Berger et al., 1996]. The spectroscopic data, ${ }^{31} \mathrm{P}\left\{{ }^{1} \mathrm{H}\right\}$ chemical shifts and ${ }^{1} J_{\mathrm{PPt}}$, for complexes 5 - $\mathbf{8}$, are listed in Table 1.
Complexes 5 and $\mathbf{6}$ exhibit the signal at 122.6 and $97.8 \mathrm{ppm}$ in the ${ }^{31} \mathrm{P}$ NMR spectrum, respectively. These peaks are shifted by $c a$. 6 and $18 \mathrm{ppm}$ to lower field relative to the signal of the free ligands indicating the $\mathrm{P} \rightarrow \mathrm{Pt}$ donation of the $\mathrm{P}^{t} \mathrm{BuCl}$ or $\mathrm{PPhCl}$ group. This effect was also observed for the platinum complexes of the related compounds, ${ }^{t} \mathrm{Bu}_{2} \mathrm{PC}_{2} \mathrm{H}_{4} \mathrm{P}^{t} \mathrm{Bu}_{2}$ [Benn et al., 1986] and $\mathrm{Ph}_{2} \mathrm{PC}_{2} \mathrm{H}_{4} \mathrm{PPh}_{2}$ [Baldwin and Fink, 2002]. Complexes $\mathbf{7}$ and $\mathbf{8}$ have, however, chemical shifts at higher field by $c a .19$ and $10 \mathrm{ppm}$ compared with the signals of the free ligands, 117.2 (3) and 104.3 ppm (4), respectively.

This effect reflects the increase in electron density at the phosphorus atoms, which is presumably due to the $\mathrm{p}_{\pi}(\mathrm{N})-\mathrm{d}_{\pi}(\mathrm{P})$ interaction. This also affects the phosphorus-platinum donor bond as well as the platinum-phosphorus backbonding, which is expected to increase as the electronegativity of the substituents at phosphorus increases [Hill et al., 1983].

The large ${ }^{1} J_{\mathrm{PPt}}$ values of the complexes $(3873-4895 \mathrm{~Hz})$ indicate the cis coordination of the bidentate phosphine ligands [Berger et al., 1996; Sturm et al., 2000; Gray et al., 2000], as shown also by the X-ray structures, whereas the ${ }^{1} J_{\mathrm{PPt}}$ values for trans platinum complexes are significantly lower [Berger et al., 1996; Johansson, et al., 2001]. While the tert-butyl groups in complex 5 cause the smallest P-Pt coupling constant, the diphenylamino groups in complex $\mathbf{8}$ are responsible for the largest P-Pt coupling constant. In general, the P-Pt coupling constants of the complexes increase with the electronegativity of the substituents on phosphorus, which increases the $\pi$-acceptor character of the phosphines [Hill et al., 1983; Grim et al., 1967].

In the ${ }^{13} \mathrm{C}$ NMR spectra, complexes 7 exhibits the complex coupling patterns of an AA'XX' spin system for the PCCP group, which appears as a pseudo-triplet, in which the coupling constants, ${ }^{1} J_{\mathrm{PC}} \cong{ }^{2} J_{\mathrm{PC}}$, is $25.2 \mathrm{~Hz}$. While a more complex pattern is observed for complex 5 due to coupling to the platinum and phosphorus atoms $\left({ }^{2} J_{\mathrm{PtC}}=102.6 \mathrm{~Hz} ;{ }^{1} J_{\mathrm{PC}}=28.1 \mathrm{~Hz}\right)$. Complex 6 is very little soluble in conventional organic solvents, therefore no ${ }^{13} \mathrm{C}$ NMR spectra could be obtained. 
Table I. ${ }^{3} \mathrm{P}\{\mathrm{I} \mathrm{H}\}$ chemical shifts, 'JPPt of complexes 5-8 and the chemical shift differences between the

\begin{tabular}{|c|c|c|c|}
\hline Complex & $\begin{array}{c}31 \mathrm{P}\{1 \mathrm{H}\} \\
(\mathrm{ppm})\end{array}$ & $\begin{array}{l}\text { 'JPPt } \\
(\mathbf{H z})\end{array}$ & $\Delta \delta(\mathrm{ppm})$ \\
\hline cis-rac- $\left[\mathrm{PtCl}_{2}\left\{\mathrm{I}, 2-(\mathrm{PtBuCl})_{2} \mathrm{C}_{2} \mathrm{~B}_{10} \mathrm{H}_{10}\right\}\right](5)$ & 122.6 & 3873 & 6 \\
\hline cis-rac- $\left[\mathrm{PtCl}_{2}\left\{\mathrm{I}, 2-(\mathrm{PPhCl})_{2} \mathrm{C}_{2} \mathrm{~B}_{10} \mathrm{H}_{10}\right\}\right](6)$ & 97.8 & 4038 & 18 \\
\hline cis-rac- $\left[\mathrm{PtCl}_{2}\left\{\mathrm{I}, 2-\left(\mathrm{P}\left\{\mathrm{NEt}_{2}\right\} \mathrm{Cl}_{2}\right)_{2} \mathrm{C}_{10} \mathrm{H}_{10}\right\}\right](\mathbf{7})$ & 98.2 & 4663 & -19 \\
\hline cis-rac- $\left[\mathrm{PtCl}_{2}\left\{\mathrm{I}, 2-\left(\mathrm{P}\left\{\mathrm{NPh}_{2}\right\} \mathrm{Cl}_{2}\right)_{2} \mathrm{C}_{2} \mathrm{~B}_{10} \mathrm{H}_{10}\right\}\right](\mathbf{8})$ & 94.6 & 4895 & -10 \\
\hline
\end{tabular}

Table II. Selected bond lengths (A) of 5 and 6

\begin{tabular}{|c|c|c|c|}
\hline \multicolumn{2}{|c|}{5} & \multicolumn{2}{|c|}{6} \\
\hline $\mathrm{Pt}(\mathrm{I})-\mathrm{P}(2)$ & $2.208(9)$ & $P t(I)-P(2)$ & $2.193(9)$ \\
\hline $\mathrm{Pt}(\mathrm{I})-\mathrm{P}(\mathrm{I})$ & $2.217(8)$ & $\operatorname{Pt}(I)-P(I)$ & $2.203(9)$ \\
\hline $\mathrm{Pt}(\mathrm{I})-\mathrm{Cl}(4)$ & $2.324(9)$ & $\mathrm{Pt}(\mathrm{I})-\mathrm{Cl}(3)$ & $2.328(I)$ \\
\hline $\mathrm{Pt}(\mathrm{I})-\mathrm{Cl}(3)$ & $2.334(9)$ & $\mathrm{Pt}(\mathrm{I})-\mathrm{Cl}(4)$ & $2.328(9)$ \\
\hline $\mathrm{Cl}(\mathrm{I})-\mathrm{P}(\mathrm{I})$ & $2.020(I)$ & $\mathrm{Cl}(\mathrm{I})-\mathrm{P}(\mathrm{I})$ & $2.007(I)$ \\
\hline $\mathrm{Cl}(2)-\mathrm{P}(2)$ & $2.008(I)$ & $\mathrm{Cl}(2)-\mathrm{P}(2)$ & $2.009(1)$ \\
\hline$P(I)-C(3)$ & I.875(3) & $P(I)-C(3)$ & I.798(4) \\
\hline$P(I)-C(I)$ & $1.890(3)$ & $P(I)-C(I)$ & $|.86|(4)$ \\
\hline$P(2)-C(2)$ & I.878(3) & $P(2)-C(9)$ & I.796(4) \\
\hline$P(2)-C(7)$ & I.88I(3) & $P(2)-C(2)$ & $1.857(4)$ \\
\hline$C(1)-C(2)$ & I.708(4) & $C(1)-C(2)$ & $1.672(5)$ \\
\hline
\end{tabular}

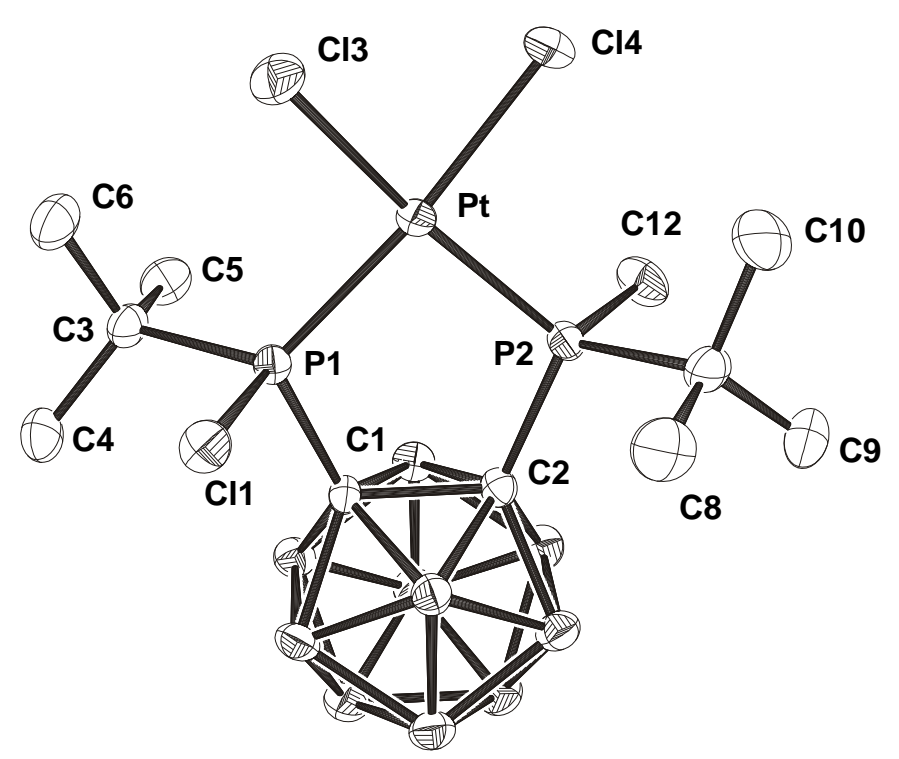

Fig. I. Molecular structure of 5 (ORTEP plot with atom labeling scheme, thermal ellipsoids are drawn at the $\mathbf{5 0} \%$ probability level, hydrogen atoms are omitted for clarity, only one enantiomer is shown) 


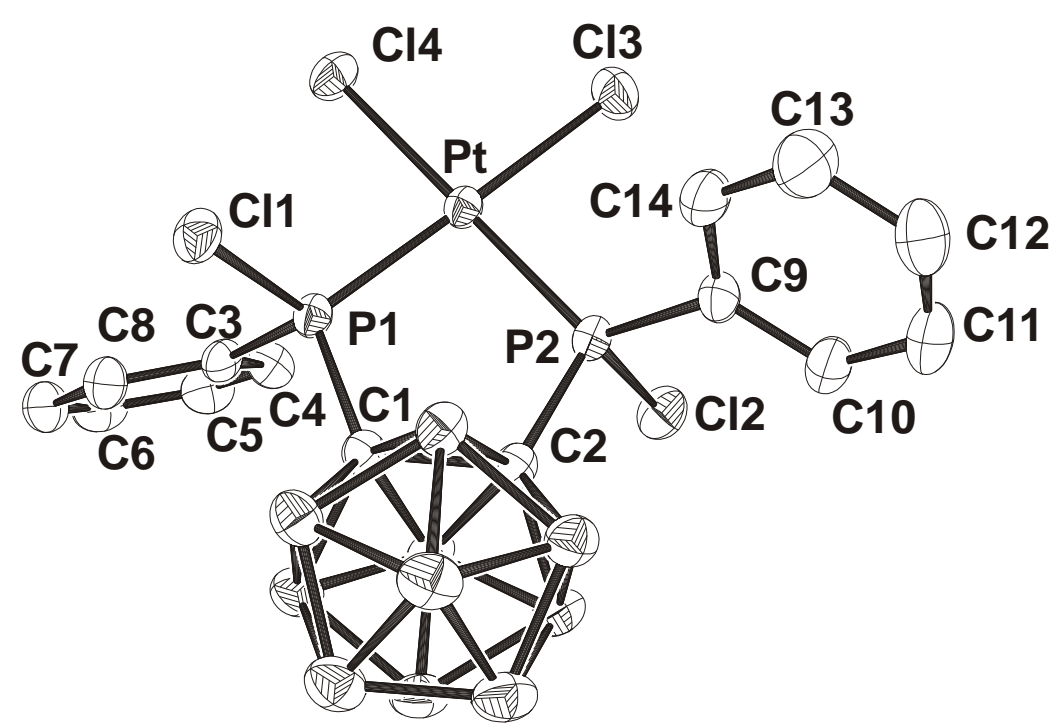

Fig. 2. Molecular structure of 6 (ORTEP plot with atom labeling scheme, thermal ellipsoids are drawn at the $\mathbf{5 0} \%$ probability level, hydrogen atoms are omitted for clarity, only one enantiomer is shown)

Table III. Selected bond angles (deg.) for 5 and 6

\begin{tabular}{|c|c|c|c|}
\hline \multicolumn{2}{|c|}{5} & \multicolumn{2}{|c|}{6} \\
\hline$P(2)-P t(I)-P(I)$ & $93.2(3)$ & $P(2)-P t(I)-P(I)$ & $92.3(3)$ \\
\hline P(2)-Pt(I)-Cl(4) & $89.5(3)$ & $\mathrm{P}(2)-\mathrm{Pt}(\mathrm{I})-\mathrm{Cl}(3)$ & $88.4(4)$ \\
\hline $\mathrm{P}(\mathrm{I})-\mathrm{Pt}(\mathrm{I})-\mathrm{Cl}(4)$ & $166.8(3)$ & $\mathrm{P}(\mathrm{I})-\mathrm{Pt}(\mathrm{I})-\mathrm{Cl}(3)$ & $178.8(3)$ \\
\hline$P(2)-P t(I)-C \mid(3)$ & $165.1(3)$ & $\mathrm{P}(2)-\mathrm{Pt}(\mathrm{I})-\mathrm{Cl}(4)$ & $178.3(3)$ \\
\hline $\mathrm{P}(\mathrm{I})-\mathrm{Pt}(\mathrm{I})-\mathrm{Cl}(3)$ & $93.0(3)$ & $\mathrm{P}(\mathrm{I})-\mathrm{Pt}(\mathrm{I})-\mathrm{Cl}(4)$ & $88.1(3)$ \\
\hline $\mathrm{Cl}(4)-\mathrm{Pt}(\mathrm{I})-\mathrm{Cl}(3)$ & $87.6(3)$ & $\mathrm{Cl}(3)-\mathrm{Pt}(\mathrm{I})-\mathrm{Cl}(4)$ & $91.2(4)$ \\
\hline$C(3)-P(I)-C(I)$ & $107.6(1)$ & $C(3)-P(I)-C(I)$ & $108.4(2)$ \\
\hline$C(3)-P(I)-C l(I)$ & $102.4(1)$ & $C(3)-P(I)-C \mid(I)$ & $104.5(I)$ \\
\hline$C(I)-P(I)-C l(I)$ & $103.6(I)$ & $C(I)-P(I)-C \mid(I)$ & $101.6(1)$ \\
\hline$C(3)-P(I)-P t(I)$ & $119.7(1)$ & $C(3)-P(I)-P t(I)$ & $117.6(1)$ \\
\hline$C(I)-P(I)-P t(I)$ & $108.8(I)$ & $C(I)-P(I)-P t(I)$ & $109.8(I)$ \\
\hline$C I(I)-P(I)-P t(I)$ & II3.5(4) & $C l(I)-P(I)-P t(I)$ & $113.6(5)$ \\
\hline$C(2)-P(2)-C(7)$ & $110.3(1)$ & $C(9)-P(2)-C(2)$ & 104.8(2) \\
\hline$C(2)-P(2)-C I(2)$ & $102.8(I)$ & $C(9)-P(2)-C I(2)$ & $105.1(1)$ \\
\hline$C(7)-P(2)-C l(2)$ & $102.0(I)$ & $C(2)-P(2)-C I(2)$ & $103.2(1)$ \\
\hline$C(2)-P(2)-P t(1)$ & $109.8(I)$ & $C(9)-P(2)-P t(1)$ & $120.3(I)$ \\
\hline$C(7)-P(2)-P t(I)$ & $116.8(1)$ & $C(2)-P(2)-P t(I)$ & 109.9(I) \\
\hline $\mathrm{Cl}(2)-\mathrm{P}(2)-\mathrm{Pt}(\mathrm{I})$ & $114.1(4)$ & $\mathrm{Cl}(2)-\mathrm{P}(2)-\mathrm{Pt}(\mathrm{I})$ & II2.2(5) \\
\hline
\end{tabular}


Table IV. Selected bond lengths $(\AA)$ and bond angles (deg.) for 7

\begin{tabular}{|c|c|c|c|}
\hline \multicolumn{2}{|c|}{ Selected bond lengths } & \multicolumn{2}{|c|}{ Selected bond angles } \\
\hline $\mathrm{Pt}(\mathrm{I})-\mathrm{P}(2)$ & $2.192(7)$ & $\mathrm{P}(2)-\mathrm{Pt}(\mathrm{I})-\mathrm{P}(\mathrm{I})$ & $92.3(2)$ \\
\hline$P t(I)-P(I)$ & $2.202(6)$ & $\mathrm{P}(2)-\mathrm{Pt}(\mathrm{I})-\mathrm{Cl}(3)$ & 179.7(3) \\
\hline $\mathrm{Pt}(\mathrm{I})-\mathrm{Cl}(3)$ & $2.342(9)$ & $\mathrm{P}(\mathrm{I})-\mathrm{Pt}(\mathrm{I})-\mathrm{Cl}(3)$ & 87.4(3) \\
\hline $\mathrm{Pt}(\mathrm{I})-\mathrm{Cl}(4)$ & $2.344(6)$ & $\mathrm{P}(2)-\mathrm{Pt}(\mathrm{I})-\mathrm{Cl}(4)$ & $88.9(2)$ \\
\hline $\mathrm{Cl}(\mathrm{I})-\mathrm{P}(\mathrm{I})$ & $2.010(8)$ & $\mathrm{P}(\mathrm{I})-\mathrm{Pt}(\mathrm{I})-\mathrm{Cl}(4)$ & $177.0(2)$ \\
\hline $\mathrm{Cl}(2)-\mathrm{P}(2)$ & $2.013(8)$ & $\mathrm{Cl}(3)-\mathrm{Pt}(\mathrm{I})-\mathrm{Cl}(4)$ & 91.4(3) \\
\hline$P(I)-N(I)$ & $1.636(2)$ & $\mathrm{N}(\mathrm{I})-\mathrm{P}(\mathrm{I})-\mathrm{C}(\mathrm{I})$ & $108.6(I)$ \\
\hline$P(I)-C(I)$ & $1.879(2)$ & $\mathrm{N}(\mathrm{I})-\mathrm{P}(\mathrm{I})-\mathrm{Cl}(\mathrm{I})$ & 107.6(9) \\
\hline$P(2)-N(2)$ & $1.628(2)$ & $\mathrm{C}(\mathrm{I})-\mathrm{P}(\mathrm{I})-\mathrm{Cl}(\mathrm{I})$ & 100.2(7) \\
\hline$P(2)-C(2)$ & $1.877(2)$ & $\mathrm{N}(\mathrm{I})-\mathrm{P}(\mathrm{I})-\mathrm{Pt}(\mathrm{I})$ & $115.0(8)$ \\
\hline \multirow[t]{8}{*}{$C(1)-C(2)$} & $1.678(3)$ & $\mathrm{C}(\mathrm{I})-\mathrm{P}(\mathrm{I})-\mathrm{Pt}(\mathrm{I})$ & $110.2(8)$ \\
\hline & & $\mathrm{Cl}(\mathrm{I})-\mathrm{P}(\mathrm{I})-\mathrm{Pt}(\mathrm{I})$ & II4.I(3) \\
\hline & & $N(2)-P(2)-C(2)$ & 107.6(I) \\
\hline & & $\mathrm{N}(2)-\mathrm{P}(2)-\mathrm{Cl}(2)$ & 106.0(9) \\
\hline & & $\mathrm{C}(2)-\mathrm{P}(2)-\mathrm{Cl}(2)$ & $100.9(7)$ \\
\hline & & $N(2)-P(2)-P t(I)$ & II8.3(8) \\
\hline & & $C(2)-P(2)-P t(I)$ & 109.9(8) \\
\hline & & $\mathrm{Cl}(2)-\mathrm{P}(2)-\mathrm{Pt}(\mathrm{I})$ & $112.8(4)$ \\
\hline
\end{tabular}

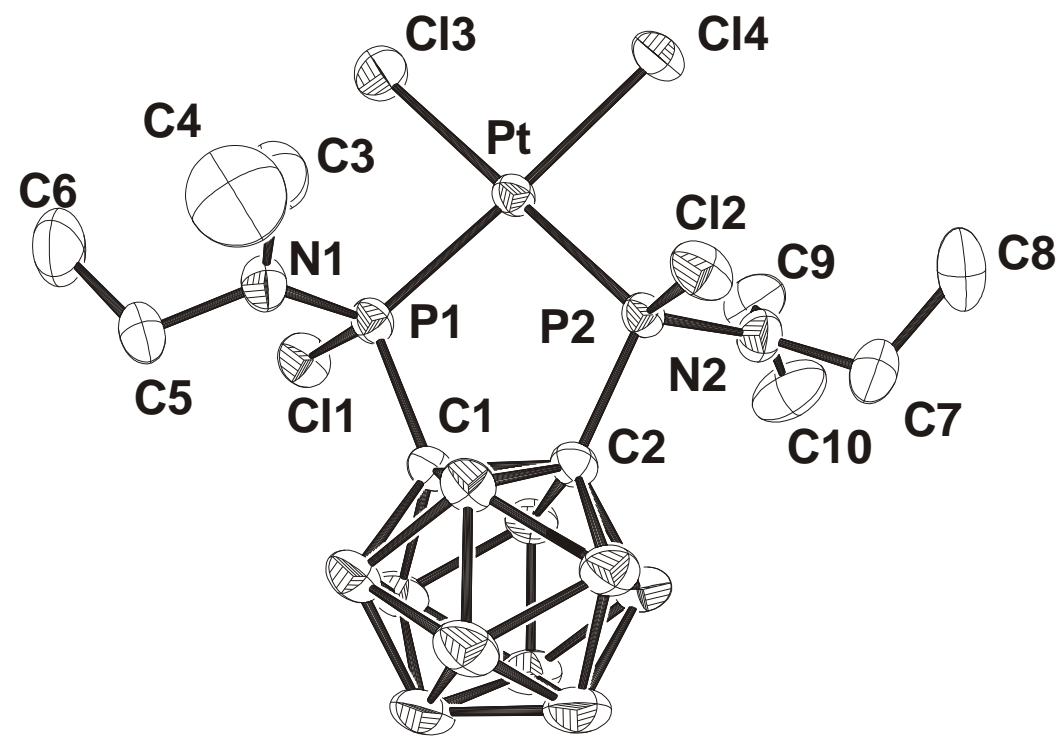

Fig. 3. Molecular structure of 7 (ORTEP plot with atom labeling scheme, thermal ellipsoids are drawn at the $\mathbf{5 0} \%$ probability level, hydrogen atoms are omitted for clarity, only one enantiomer is shown) 
The carbon atoms of the carbaborane cluster in 7 also show coupling to the platinum atom $\left({ }^{2} J_{\mathrm{PtC}}\right.$ $=169 \mathrm{~Hz}$ ), whereas no coupling was observed in other platinum complexes. The platinum-carbon coupling was observed also for the tert-butyl group of compound $5\left({ }^{2} J_{\mathrm{PtC}}=54 \mathrm{~Hz}\right)$.

The chemical shift of the carbon atoms of the tert-butyl group in compound $\mathbf{1 0}$ is shifted downfield by $c a .10 \mathrm{ppm}$ with respect to that in the free ligand $\mathbf{1}$ [Sterzik et al., 2001] and split into a triplet of pseudotriplets due to coupling with the platinum and phosphorus atoms $\left({ }^{1} J_{\mathrm{PC}}=40.1\right.$ and ${ }^{3} J_{\mathrm{PC}}=7.9 \mathrm{~Hz}$ ), whereas in the free ligand the coupling constant is smaller $\left({ }^{1} J_{\mathrm{PC}}=17 \mathrm{~Hz}\right)$.

Single crystals of 5 and $\mathbf{6}$ were obtained from a concentrated toluene solution at room temperature. Compound $\mathbf{5}$ crystallizes in the orthorhombic space group Pbca with eight formula units in the unit cell, complex 6 crystallizes in the triclinic space group $P \overline{1}$ with two molecules in the unit cell. Due to the crystallographic center of inversion, both enantiomers $(R, R$ and $S, S)$ are present in the unit cell of compound 5 and $\mathbf{6}$ (Fig. 1 and Fig. 2). Selected bond lengths and angles of 5 and $\mathbf{6}$ are reported in Table 2.

Colorless crystals of compounds 7 was obtained from a toluene solution at room temperature. Single crystals of $\mathbf{7}$ crystallizes in the triclinic space group $P \overline{1}$, in which two molecules of 7 and one molecule of toluene were found in the unit cell of 7. Selected bond lengths and angles of 7 are collected in Table 4.

The structure of the platinum complexes consists of a slightly distorted square-planar coordination sphere, in which the platinum atom is bonded to two chlorides and two phosphorus atoms of the chelating carbaboranylphosphine. Thus the platinum atoms exhibit the coordination number four, which is preferred in platinum(II) complexes [Paavola et al., 2002; Hill et al., 1983; Cravotto et al., 2005; Hush et al., 2005]. The sums of the bond angles of platinum are around $360^{\circ}$. The coordination of the chelating carbaboranylphosphine ligands to platinum leads to the formation of a five-membered ring.

As expected, the $\mathrm{P}(1)-\mathrm{Pt}-\mathrm{P}(2)$ bond angles in the monomer platinum complexes are found between $92.3(3)^{\circ}\left(\mathbf{6}\right.$ and 7) and 93.2 ${ }^{\circ}$ (3) (5), which is the preferred P-M-P bite angle for square-planar complexes with two carbon atoms as spacer between the two phosphorus donor atoms [Van Leeuwen et al., 2000]. According to the Cambridge Crystallographic Data Center (CCDC) the P-Pt-P bond angles for related platinum complexes lie in the range of 82.4 [Vasconselos et al., 1998] to $93.1^{\circ}$ [Paavola et al., 2002]. Thus, compound 5 exhibits the largest P-Pt-P bond angle.

The Pt-P distances in 7 is 2.192(7) $\AA$, which is in the range of Pt-P bond lengths for related compounds (2.182 $\AA$ [Claver et al., 2000] - 2.278 $\AA$ [Harada, 1976]). The short bond lengths indicate relative strong Pt-P bonds, which can be rationalized by the presence of the orthocarbaborane backbone as well as the electronwithdrawing substituents on the phosphorus atoms. The Pt-Cl bonds in 5 is 2.324(9) $\AA$, which is in agreement with those observed in related platinum complexes (2.327-2.395 $\AA$ [Dahlenburg and Mertel, 2001]).

It has been reported previously, that the P-Pt coupling constants reflect the strength of the Pt-P bonds [Hill et al., 1983]. Therefore, compound 7, which possess the largest ${ }^{1} J_{\mathrm{PPt}}$ coupling constant, display the shortest Pt-P bonds. This trend is expected since the electron-withdrawing substituents on the phosphorus atoms increase the $\mathrm{d}_{\pi} \rightarrow \mathrm{d}_{\pi}$ interaction between the platinum and the phosphorus donor atoms [Grim et al., 1967]. On the other hand, this so-called backbonding would also increase the $\sigma$-bond character of the Pt-P bond by a synergistic effect [Grim et al., 1967]. The backbonding effect is also revealed by the ${ }^{31} \mathrm{P}$ NMR spectrum, in which the signals of the platinum complexes $\mathbf{7}$ - $\mathbf{8}$ are shifted to higher field relative to those of the free ligands.

The C-C distances of the carbaborane cage were found to decrease on complexation to platinum. Compound $\mathbf{5}$ provides the largest decrease in $\mathrm{C}-\mathrm{C}$ distance for the mononuclear platinum complexes, i.e. $0.062 \AA$ relative to that of the free ligand 1 (1.770 $\AA$ ) [Sterzik et al., 2001]. The diminution of C-C distances can be rationalized by the change of electronic properties of the phosphorus atoms due to complexation.

It was observed that major elongation of the $\mathrm{C}-\mathrm{C}$ bond is obtained when the element with the lone pair of electrons is directly connected with the cluster. The electron density from the available lone pair of electrons of the element is transferred to the cage, producing an increase in the C-C distance [Teixidor et al., 2003]. The electron density on the phosphorus atoms in $\mathbf{5}$ is lower due to the strong $\sigma(\mathrm{P} \rightarrow \mathrm{Pt})$ donor character of the $\mathrm{P}^{t} \mathrm{BuCl}$ group, resulting in a larger decrease in $\mathrm{C}-\mathrm{C}$ bond length relative to the free ligand.

A decrease in $\mathrm{P}_{-} \mathrm{C}_{\text {cluster }}$ bond lengths is also observed for the the platinum complexes, which is probably due to the change in electron density of the phosphorus atoms during the complexation. Compound $\mathbf{6}$ shows the largest decrease in the P- 
$\mathrm{C}_{\text {cluster }}$ distance of the mononuclear platinum complexes, which indicates a higher electron density on the phosphorus atoms.

\section{CONCLUSION}

Compounds 1, 2, 3, and 4 show the capability to act as ligands in complexation reactions with platinum metal. The platinum complexes cis-rac- $\left[\mathrm{PtCl}_{2}\left\{1,2-(\mathrm{PRCl})_{2} \mathrm{C}_{2} \mathrm{~B}_{10} \mathrm{H}_{10}\right\}\right]$ $\left(\mathrm{R}={ }^{t} \mathrm{Bu}(5), \mathrm{Ph}(\mathbf{6}), \mathrm{NEt}_{2}(\mathbf{7}), \mathrm{NPh}_{2}(\mathbf{8})\right.$ were obtained from the reaction of $\left[\mathrm{PtCl}_{2}(\mathrm{COD})\right]$ with the corresponding chiral carbaboranylphosphine ligand $1-4$. The complexes mentioned above were fully characterized by NMR, IR, and MS, and, other than $\mathbf{1 4}$ and 22, also by X-ray crystallography.

\section{ACKNOWLEDGMENT}

We gratefully acknowledge support from the Deutscher Akademischer Austausch Dienst (DAAD doctoral grant for I.M.).

\section{REFERENCES}

Alexander, R.P. and Schroeder, H.J., 1963, Chemistry of Decaborane-Phosphorus Compounds. IV. Monomeric, Oligomeric, and Cyclic phosphino-carboranesl, Inorg. Chem., 2, II 07.

Balema, V.P., Blaurock, S. and Hey-Hawkins, E., 1999, The reaction of phenylchloro phosphino substituted dicarba-closododecaboranes(I2) with elemental sulfur and molecular structures of rac-bisphenylchlorophosphino dicarba-closododecaborane and phenylchlorothiophosphino phenyldicarbacloso-dodecaborane, Polyhedron, I 8, 545.

Bakun, A.V., Yu. P. Dokuchaev, I. I. Lapidis, Yu. V. Moskovskii, I. M. Skvortsov, B. G. Antokin, USSR Patent, 40224I.

Berger, S., Braun, S. and Kalinowski, H.-O., 1996, NMR-Spectroscopy of the Non-Metallic Elements, $\mathrm{VCH}$ Verlagsgesellschaft $\mathrm{mbH}$, Weinheim.

Benn, R., Jolly, P.W., Joswig, T., Maynott, R. And Schick, K.-P., 1986, Z. Naturforsch., 4lb, 680.

Brauer, G., 198I, Handbuch der Präparativen Anorganischen Chemie, Band III, Ferdinand Enke Verlag, Stuttgart.

Claver, C., Fernandez, E., Gillon, A., Heslop, K., Hyett, D.J., Martorell, A., Orpen, A.G. and
Pringle, P.G., 2000, Biarylphosphonites: a class of monodentate phosphorus(III) ligands that outperform their chelating analogues in asymmetric hydrogenation catalysis, Chem. Comm., 961.

Cravotto, G., F. Demartin, G. Palmisano, A. Penoni, T. Radice, S. Tollari, 2005, Novel cyclometallated $\mathrm{Pd}(\mathrm{II})$ and $\mathrm{Pt}$ (II) complexes with indole derivatives and their use as catalysts in Heck reaction, J. Organomet. Chem., 690, 2017.

Cotton, F.A., 1972, Inorg. Syn., 13, 48.

Dahlenburg, L., S. Mertel, 200I, Chirale Bisphosphane VI. Chelatkomplexe von Platin(II) mit racemischen und optisch reinen trans-Cyclopentan-I,2-diylbis(phosphonigsa urediester)- und transCyclopentan-I,2-diyl-

bis(phosphonigsa urediamid)-Liganden: Synthesen, Strukturen und $\mathrm{C}-\mathrm{H}-$ Aktivierungsreaktionen, J. Organomet. Chem., 630, 22I Falius, H., M. Babin, 1976, Z. Anorg. Allg. Chem., 420, 65.

Gray, G. M., D. C. Smith Jr., C. H. Duffey, 2000, ${ }^{31} \mathrm{P}\{\mathrm{IH}\}$ NMR spectroscopic and $X$-ray crystallographic studies of conformational dynamics in cis$\mathrm{M}\left\{\mathrm{Ph}_{2} \mathrm{P}\left(\mathrm{CH}_{2} \mathrm{CH}_{2} \mathrm{O}\right)_{5} \mathrm{CH}_{2} \mathrm{CH}_{2} \mathrm{PPh}_{2}-\mathrm{P}, \mathrm{P}\right\}$ ( $\mathrm{M}$ - Mo $(\mathrm{CO})_{4}, \mathrm{PtCl}_{2}$ ) metallacrown ethers, Inorg. Chem., 58I, 300-302.

Grim, S. O., R. L. Keiter, W. McFarlane, 1967, A Phosphorus-3I Nuclear Magnetic Resonance Study of Tertiary Phosphine Complexes of Platinum( II), Inorg. Chem., 6, II33.

Harada, M., Y. Kai, N. Yasuoka, N. Kasai, 1976, Bull. Chem. Soc. Japan., 49, 3472.

Hawthorne, M.F., 1993, Angew. Chem. 105, 997.

Hill, W. E., B. G. Rackley, L. Silva-Trivino, 1983, Inorg. Chem. Acta, 75, $5 \mathrm{I}$.

Hush, N. S., J. Schamberger, G. B. Bacskay, 2005, A quantum chemical computational study of the relative stabilities of cis- and transplatinum dichloride in aqueous solution, Coord. Chem. Rev., 249, 299.

Van Leeuwen, P. W. N. M., P. C. J. Kamer, J. N. H. Reek, P. Dierkes, 2000, Ligand Bite Angle Effects in Metal-catalyzed C-C Bond Formation, Chem. Rev., 100, 274I.

Imori, T., T. Ninomiya, K. Konda, 1992, Jpn. Kokai Tokkyo Koho, 4.

Johansson, M. H., T. Malmström, O. F. Wendt, 200I, Chiral platinum(II) complexes. Crystal and molecular structures of cis$[\mathrm{PtPhCl}((R, R)-\mathrm{CHIRAPHOS})]$ and cis- 
$\left[\mathrm{PtCl}_{2}((R, R)-\mathrm{CHIRAPHOS})\right]$ Inorg. Chim. Acta, 316, 149.

Kopf-Maier, 1994, Complexes of metals other than platinum as antitumour agents, Eur. J. Clin. Pharmacol, 47, I.

Larsen, A. S., J. D. Holbrey, F. S. Tham, C. A. Reed, 2000, Designing lonic Liquids: Imidazolium Melts with Inert Carborane Anions, J. Am. Chem. Soc., I 22, 7264.

Leites, L.A., 1992, Vibrational Spectroscopy of Carboranes and Parent Boranes and Its Capabilities in Carborane Chemistry, Chem. Rev., 92, 279.

Locher, G.L., 1936, Am. J. Roentgenol. Radium Ther., 36, I.

Longato, B. and Bresadola, S., 1982, Hydridoalkyland Hydridoalkenyliridium( III) Complexes Formed in the Reaction of (Carboranyl)iridium(III) Dihydrides with Alkenes and Alkynes. I, Inorg. Chem., 2I, 168.

Paavola, S., Teixidor, F., Viñas, C. and Kivekäs, R., 2002, Pd(II) bromide complexes of I,2bis(diphenylphosphino)-I,2-dicarbaclosododecaborane. Crystal structures of $\left[\mathrm{PdBr}_{2}\left(\mathrm{I}, 2-\left(\mathrm{PPh}_{2}\right)_{2}-\mathrm{I}, 2-\mathrm{C}_{2} \mathrm{~B}_{10} \mathrm{H}_{10}\right)\right] \mathrm{CH}_{2} \mathrm{Cl}_{2}$, $\left[\mathrm{PdBr}_{1.133} \mathrm{Cl}_{0.867}\left(\mathrm{I}, 2-\left(\mathrm{PPh}_{2}\right)_{2}-\mathrm{I}, 2-\mathrm{C}_{2} \mathrm{~B}_{10} \mathrm{H}_{10}\right)\right]$ $\mathrm{CH}_{2} \mathrm{Cl}_{2}$ and $\left[\mathrm{PdBrC}_{10.541} \mathrm{Me}_{0.459}\left(\mathrm{I}, 2-\left(\mathrm{PPh}_{2}\right)_{2}-\right.\right.$ I,2- $\left.\left.\mathrm{C}_{2} \mathrm{~B}_{10} \mathrm{H}_{10}\right)\right] \mathrm{CHCl}_{3}$, Acta Crystallogr., C58, $\mathrm{m} 237$.

Peyrone, M., 1844, Annalen der Chemie und Pharmacie, Band LI, I ff.

Perich, J.W. and Johns, R.B., 1988, Synthesis, 2, 142

Rosenberg, B., VanCamp, L., Trosko, J.E. and Mansour, V.H., 1969, Nature, 222, 385.

Rozencweig, M., von Hoff, D.D. and Slovik, M., 1987, Cis-Diamminochloroplatinum, Ann. Intern. Med., 86, 803.

SHELXTL PLUS, SHELXS, 1990, Program for Crystal Structure Solution, SHELXL, Program for Crystal Structure Determination, XP, Interactive Molecular Graphics, Siemens Analytical X-ray Institute Inc.

Sheldrick, G.M., 1998, SADABS - Program for Empirical Absorption Correction, Göttingen.

Soloway, A.H., Tjarks, W., Baruum, B.A., Rong, F.G., Barth, R.F., Codogni, I.M. and Wilson, J.G., 1998, The Chemistry of Neutron Capture Therapy, Chem. Rev., 98, I5I5.

Sterzik, A., Rys, E., Blaurock, S. and Hey-Hawkins, E., 200I, Synthesis and coordination properties of I-tert-butylchlorophosphinoand I,2-bis(tert-butylchlorophosphino)-I,2-
dicarba-closo-dodecaborane(I2)-

molecular structures of rac and meso-I,2$\left(\mathrm{P}^{t} \mathrm{BuCl}\right)_{2} \mathrm{C}_{2} \mathrm{~B}_{10} \mathrm{H}_{10}$ and $(R, R, R, R / S, S, S, S)$ $\left[\left\{\mathrm{Cu}\left\{\mathrm{I}, 2-\left(\mathrm{P}^{\mathrm{t} B u C l}\right)_{2} \mathrm{C}_{2} \mathrm{~B}_{10} \mathrm{H}_{10}\right\}(\mathrm{Cl})\right\}_{2}\right]$, Polyhedron, 20, 3007.

Stadlbauer, S., Frank, R., Maulana, I., Loennecke, P., Kirchner, B. and Hey-Hawkins, E., 2009, Synthesis and Reactivity of orthoCarbaborane-Containing Chiral Aminohalophosphines, Inorg. Chem., 48, 6072-6082.

Sturm, T., Weissensteiner, W., Mereiter, K. Kégl, T., Jeges, G., Petölz, G. and Kollár, L., 2000, Platinum complexes of heteroannularly bridged heterobidentate ferrocenyl diphosphine ligands: their molecular structure and their use in catalytic carbonylation reactions, J. Organomet. Chem., 595, 93.

Teixidor, F., Viñas, C., Demonceau, A. and Nuñez, R., 2003, Boron clusters: Do they receive the deserved interest?, Pure Appl. Chem., 75, 1305.

Teixidor, F., Flores, M.A., Viñas, C., Kivekäs, R. and Sillanpää, R., 1996, Rhodium Complexes with the New Anionic Diphosphine [7,8-( $\left.\left.\mathrm{PPh}_{2}\right)_{2}-7,8-\mathrm{C}_{2} \mathrm{~B}_{9} \mathrm{H}_{10}\right]-$ Ligand, Angew. Chem. Int. Ed. Engl., 35, 225I.

Valliant, J.F., Guenther, K.J., King, A.S., Morel, P. Schaffer, P., Sogbein, O.O. and Stephenson, K.A., 2002, The medicinal chemistry of carboranes, Coord. Chem. Rev., 232, 173.

Vasconselos, I.C.F., Anderson, G.K., Rath, N.P. and Spilling, C.D., 1998, Preparation and Structure of new homochiral diazaphosphol ligands and their platinum(II) chloride complexes, Tetrahedron: Asymmetry, 9, 927.

Williams, R.E., 1992, The Polyborane, Carborane, Carbocation Continuum: Architectural Patterns, Chem. Rev., 92, 177.

Yinghuai, Z., Parthiban, A. and Kooli, F.B.H., 2004, Syntheses and catalytic properties of polystyrene supported nickel(II) carborane complexes, Catalysis Today, 96, I43. 